\title{
Documentos
}

\section{EPILEPSIA Y ANTICONCEPTIVOS}

\author{
Nelson Burgos S. ${ }^{1}$, Manuel Parra A. ${ }^{1}$ \\ ${ }^{1}$ Departamento de Obstetricia y Ginecología, Hospital Clínico J. J. Aguirre, Universidad de Chile
}

\section{RESUMEN}

La epilepsia es una enfermedad neurológica que puede afectar a la mujer en edad fértil. Es conocido que las terapias anticonvulsivantes pueden disminuir la efectividad de los anticonceptivos hormonales. Los principales mecanismos son una mayor estimulación del sistema enzimático hepático de la citocromo P450 y el aumento de los niveles de la globulina transportadora de hormonas sexuales. Como consecuencia se produce una disminución de los niveles disponibles de los anticonceptivos hormonales, debiendo ajustar las dosis y en algunas oportunidades utilizar otros métodos.

\section{PALABRAS CLAVES: Epilepsia, anticonvulsivantes, anticonceptivos orales, citocromo P450, globulina transportadora de hormonas sexuales}

\section{SUMMARY}

Epilepsy is a neurological disease that can affect to the woman in fertile age. It is known that the anticonvulsive therapies can diminish the effectiveness of the hormonal contraceptives. The main mechanisms are a greater stimulation of the hepatic enzymatic system of cytochrome P450 and the higher levels of the sexual hormone transporting globulin. As consequence takes place a diminution of the levels available of the hormonal contraceptives, having to fit the doses and in some opportunities to use other methods.

\section{KEY WORDS: Epilepsy, anticonvulsants, oral contraceptives, cytochrome P450, sexual hormone transporting globulin}

\section{INTRODUCCIÓN}

La epilepsia es uno de los desordenes más comunes y desafiantes de las enfermedades neurológicas que afectan a niños y adultos. Existiendo diferentes modalidades para su tratamiento, son los medicamentos antiepilépticos los que obtienen mejores resultados con una mejor calidad de vida $(1,2)$. El manejo clínico de la epilepsia en la mujer ha debido tomar en consideración una variedad de factores del ámbito social y cultural, como la edad, diagnóstico y caracterización de la epilepsia, los síndromes femeninos específicos, la influencia de las hormonas femeninas, como también el uso de anticonceptivos hormonales y la terapia de reemplazo hormonal (2). Alrededor de 65 millones de mujeres en el mundo y alrededor de 14 millones en EUA usan anticonceptivos hormonales orales (ACO), siendo la píldora altamente efectiva en la contracepción con una tasa de falla menor a 1 embarazo por 100 años mujer en estudios clínicos con esquemas combinados y aproximadamente 1-3 embarazos por 100 años mujer en la población general. La falla del método puede ser atribuida a un bajo cumplimiento de las indicaciones o a la interacción con otras drogas.

Con el transcurrir del tiempo la tendencia en anticoncepción hormonal ha sido reducir las dosis de los diferentes preparados anticonceptivos, con el propósito de disminuir el riesgo de enfermedad 
tromboembólica y otras potenciales complicaciones $(3,4,5)$. Al considerar la calidad de vida de la mujer con epilepsia se debe tener claro cuáles son los métodos de control de la natalidad que se pueden utilizar en estas pacientes y cuáles son las consideraciones a tomar en cuenta al utilizar la alternativa de los ACO u otros de tipo hormonal (2). Los cuales, en caso de ser indicados deben considerar la droga antiepiléptica utilizada y la capacidad de fertilidad de la paciente. Tomando en consideración la posible falla del método y los efectos adversos (2). En 1995 en el Reino Unido y Gales $16,7 \%$ de las mujeres con epilepsia entre 15-45 años tomaban ACO comparado al $25 \%$ de la población general y solo $43,5 \%$ usaban preparados con $50 \mu \mathrm{g}$ de etinilestradiol o más (6).

En un estudio conducido por la Asociación Británica de la Epilepsia, $51 \%$ de las mujeres con epilepsia deseaban saber la interacción entre los ACO y los anticonvulsivantes y $34 \%$ de ellas había discutido con su médico la posibilidad de un embarazo (7). En Estados Unidos solo 4\% de los neurólogos y ningún ginecólogo pudo describir los efectos de los seis anticonvulsivantes más comunes en uso y alrededor del $25 \%$ reportó embarazos inexplicables en sus pacientes epilépticas con uso de algún ACO (7).

Es sabido que los ACO tienen mayor tendencia a fallar en mujeres que usan drogas antiepilépticas. Es también conocido que los estrógenos bajan el umbral de las convulsiones, aumentando la excitabilidad del SNC, pero no se ha demostrado que aumenten el riesgo de epilepsia en mujeres no afectadas o que aumenten la frecuencia de las convulsiones en las que ya tienen la enfermedad $(3,4,8,9,10,11,12)$. También ha sido sugerido que el componente progestágeno puede tener un efecto contrario contrabalanceado al componente estrogénico en la aparición de convulsiones, dándole un factor protector $(3,4,10)$. En los textos para el médico aprobados por las autoridades sanitarias se establece: 1) los ACO deberían ser suspendidos si la mujer con epilepsia experimenta un aumento de las convulsiones y 2) la aparición de epilepsia durante la toma de ACO puede indicar que este debe ser discontinuada $(8,13)$.

El uso de ACO en las mujeres con epilepsia presenta dos preguntas de importancia, parcialmente respondidas hasta hoy. Primero, ¿el uso de anticonceptivos altera la frecuencia o severidad de las convulsiones? Segundo, ¿son los ACO efectivos en la prevención de la concepción? (11). El propósito del artículo es poder responder principalmente a la segunda pregunta.

\section{FISIOLOGÍA Y MECANISMOS DE INTERACCIÓN}

El efecto de las hormonas de los ACO en la frecuencia de las convulsiones. A pesar del bien conocido efecto de los estrógenos en bajar el umbral convulsivante, nadie ha podido demostrar que los ACO que contengan estrógenos empeoren la frecuencia de las convulsiones. Además es conocido que la progesterona en forma experimental las inhibe lo que en teoría debería contrarrestar la acción convulsivante de los estrógenos, logrando un balance $(3,11)$.

Las tres principales vías más utilizadas en la administración de los anticonceptivos hormonales a saber orales, implantes subcutáneos e inyectables pueden ser adversamente impactados por los anticonvulsivantes (14).

Existen dos mecanismos que pueden explicar la falla de los ACO en las mujeres epilépticas: en primer lugar la inducción por parte de los anticonvulsivantes de las enzimas microsomales hepáticas, y en segundo lugar el incremento de la capacidad de las globulinas transportadoras de hormonas sexuales (SHBG) $(3,15,16)$.

Los esteroides son susceptibles de interactuar con varias drogas. Son metabolizados por el hígado, altamente unidos a proteínas (fracción libre menor al $2 \%$ ) y tienen una baja y variable biodisponibilidad $(3,10,11)$. Es bien conocido el efecto de algunos anticonvulsivantes en la inducción de hormonas sexuales endógenas y exógenas, como estradiol y progesterona, lo que podría explicar la falla de los ACO en las mujeres que usan concomitantemente anticonvulsivantes, los que bajan la concentración de estrógenos hasta 40$50 \%$, pero nada ha sido directamente probado $(3$, $11,14,16)$.

Por otro lado la falla de los ACO no ha sido reportada en mujeres que toman anticonvulsivantes que no inducen las enzimas hepáticas (ej. lamotrigina, gabapentina, ácido valproico), apoyando fuertemente el argumento. Por otro lado la fenitoína, fenobarbital, primidona, etosuximida y otros nuevos como el topiramato, son inductores de la citocromo P-450 que es parte del sistema enzimático de oxigenasas hepáticas microsomales $(3,4,5,7,11,14,16)$. La inducción de estos anticonvulsivantes se caracteriza por la proliferación del retículo endoplásmico del hepatocito rico en oxidoreductasas, esto conduce a una aceleración del catabolismo endógeno y exógeno de esteroides hormonales y en algunos casos de la falla de los ACO (5). Este sistema enzimático es el mayor responsable de la biotransformación de los 
anticonvulsivantes que pasan por el metabolismo hepático, el que crea una carga polar positiva que lo hace un compuesto soluble en agua y para así poder ser excretado por el riñón $(3,17)$. La tasa de metabolismo hepático depende de la fracción libre de la droga, la fracción de extracción, el flujo sanguíneo hepático y las propiedades intrínsecas de la enzima. Este sistema puede estar influido por la edad, sexo, variaciones genéticas, estado hormonal, enfermedades concurrentes y exposición a ciertas drogas o químicos $(3,15)$. El principal mecanismo es la hidroxilación-2 dependiente del citocromo P-450, para formar el 2-hidroxietinilestradiol. Hay una variedad de isoenzimas en el sistema que están bajo control genético individual que catalizan esta reacción (18). Por ejemplo la conversión de la carbamazepina a su principal metabolito el 10,11-epóxido es conducido por la isoenzima CYP3A4, la fenitoína es metabolizada por la CYP2C9 y la CYP2C19 y el fenobarbital por la CYPA3A4 y la CPY2E1. Es importante destacar que la isoenzima CYP3A4 es la principal responsable de la metabolización de las hormonas sexuales femeninas. La inducción enzimática del anticonvulsivante es dosis dependiente e involucra el tiempo de síntesis para la nueva enzima $(3,11$, $15,17)$. Diferentes agentes inductores afectan distintas isoenzimas individuales.

Como segundo mecanismo se produce un incremento de la síntesis hepática de la SHBG. Esto lleva a una disminución de la fracción libre de la hormona, teniendo mayor importancia para los progestágenos, reduciendo así la efectividad del ACO. Lo que se refleja en el sangrado a mitad de ciclo o spotting, siendo este un signo frecuente de niveles hormonales inadecuados y riesgo de inefectividad de los ACO, reduciendo por tanto su capacidad protectora $(3,5,7,11,14,15)$. Sin embargo, teóricamente la actividad farmacológica de concentración libre de progestágenos permanece constante $(15,19)$. Se ha demostrado experimentalmente que algunos compuestos de los anticonvulsivantes pueden provocar una disrupción en la hemostasia endocrina, cambiando la distribución de algunos elementos y de la expresión del mRNA de las hormonas. Los anticonvulsivantes también pueden influir en la conjugación sulfato y la glucuronización de las hormonas sexuales en la pared del intestino e hígado respectivamente $(5,11,17)$. Ha sido reportado que la tirosina-fosfatasa-gamma se relaciona con un aumento de la expresión de receptores de estrógenos alfa y que jugaría un papel en la regulación de los estrógenos (2).

\section{CLÍNICA}

Es sabido que las mujeres con epilepsia tienen de un tercio a un cuarto menos embarazos que la población general, esto puede ser explicado por un fenómeno social u orgánico (14). Socialmente un estudio en Montreal demostró que las pacientes con epilepsia se casaban menos. Además las mujeres con epilepsia según un estudio de Schupf et al aumentaban su tasa de fertilidad desde $36 \%$ al estar soltera a solamente $42 \%$ al estar casadas. Pacientes epilépticas, tendrían mayor tendencia al síndrome de ovario poliquístico y al hipoganodismo hiper o hipogonadotrófico, además de variaciones en las concentraciones y pulsos de LH, situaciones que se pueden ver aumentadas con el uso de algunos anticonvulsivantes. También se ha demostrado que las convulsiones aumentan los niveles de prolactina y FSH (14). La tasa de embarazos de 1-3 por 100 años mujer de la población general usuaria de ACO, se ve aumentada en las usuarias de anticonvulsivantes. Anecdóticamente en 1970 fueron reportados algunos casos de embarazo en pacientes con ACO y que usaban anticonvulsivantes. Coulam y Annegers describieron 25 casos en mujeres que usaron fenitoína o barbitúricos y 2 casos en usuarias de carbamazepina (4). Interesantemente todos los casos usaban dosis altas de estrógeno (50-100 $\mu \mathrm{g})$. Los autores observaron que la falla fue de 25 veces mayor para las que tomaban ACO y anticonvulsivantes que para las que tomaban solo ACO, siendo los grupos comparables, no fallando en el grupo de ACO solo. En el grupo con ACO las crisis convulsivas fueron bien controladas $(3,4,5,7)$.

En un estudio de adolescentes que usaban ACO y anticonvulsivantes con dosis en general menor a las recomendadas en los ACO (16), se incrementaron las irregularidades menstruales, alcanzando a un $64 \%$ en el grupo mencionado y solo de $9 \%$ y $5 \%$ para los grupos con ACO solo y anticonvulsivantes solos respectivamente; para la presencia de spotting no hubo diferencias importantes aunque era mayor para las con ACO solo. La irregularidad menstrual se controló aumentando la dosis de estrógenos en la píldora. Al subir la dosis del anticonvulsivante sobre todo de fenobarbital se observó un aumento de las irregularidades menstruales, no observándose embarazos.

Al revisar cada uno de los anticonvulsivantes por separado en relación al uso de ACO se observa que:

1) Fenobarbital: Existen casos anecdóticos. En un estudio de los años 80 por Back et al (20), se 
estudió cuatro mujeres que eran usuarias de ACO y luego desarrollaron epilepsia. Al estudiar la concentración de etinilestradiol y noretisterona antes y durante el uso de fenobarbital, estos bajaron su concentración en dos de las pacientes y en una se manifestó con sangrado en la mitad del ciclo, lo que se interpretó como falla incipiente del ACO. Además notaron que en 7 de 11 pacientes que recibieron fenobarbital como monoterapia y ACO tenían sangrado intermenstrual (20).

2) Fenitoína, Carbamazepina y Acido Valproico: En estudios utilizando preparados con etinilestradiol $50 \mu \mathrm{g}$ y levonogestrel $250 \mu \mathrm{g}$ antes y durante el uso de carbamazepina, fenitoína y ácido valproico, las pacientes en tratamiento con fenitoína y carbamazepina mostraron una significativa reducción de hasta un $50 \%$ en la concentración de etinilestradiol y levonogestrel $(4,11,15)$. Sin embargo no hubo diferencias significativas para las pacientes en tratamiento con ácido valproico. Interesantemente, el peak de concentración del etinilestradiol fue más alta con el ácido valproico, lo que teóricamente debería incrementar la eficacia de la anticoncepción $(5,15,21,22)$. Para Sonnen en un estudio de 32 pacientes, este observó que las pacientes que usaron ácido valproico por un período de 4-39 meses no presentaron episodios de sangrado. En cuatro de seis pacientes con carbamazepina y una de dos pacientes con fenitoína hubo sangrado intermenstrual $(15,23)$.

3) Etosuximida: Se han reportado embarazos en pacientes que toman etosuximida más ACO. Aunque las suximidas no tienen propiedades de inducción enzimática, no es claro cual puede ser su mecanismo de interacción con los ACO (15).

4) Clobazam, Clonazepam y Diazepam: Los efectos del clobazam no están estudiados, pero sería una droga que inhibe el sistema enzimático CYP. Para clonazepam y diazepam no hay interacción (15).

5) Vigabatrina: Un estudio realizado en 13 voluntarias, usando vigabatrina con $\mathrm{ACO}$ en base a $30 \mu \mathrm{g}$. etinilestradiol y $150 \mu \mathrm{g}$. levonogestrel, no hubo diferencia significativa en los parámetros farmacocinéticos. El estudio concluye que no hay interacción entre vigabatrina y los ACO combinados $(15,24)$.

6) Lamotrigina: En estudios con uso de $30 \mu \mathrm{g}$ de etinilestradiol y $150 \mu \mathrm{g}$ de levonogestrel, se observó una leve disminución en la concentración de etinilestradiol y el levonogestrel no mostró cambios. Por lo tanto la lamotrigina no afectaría la concentración plasmática de los ACO combinados $(15,25)$.
7) Gabapentina: Esta no es metabolizada y es excretada sin cambios por la orina. Por lo que no tendría efecto enzimático a nivel hepático, un estudio demostró que usando $50 \mu \mathrm{g}$ de etinilestradiol y $2,5 \mathrm{mg}$ de noretisterona, la gabapentina produjo pequeños e insignificantes incrementos en el etinilestradiol. Por lo que parece no reducir el efecto de los ACO $(15,26)$.

8) Topiramato: En estudios in vitro, parece no tener un efecto significativo en las enzimas microsomales del hígado. Pero otros estudios demostraron que puede inhibir significativamente la CYP2C19. Al combinar uso con $35 \mu \mathrm{g}$ etinilestradiol y $1 \mathrm{mg}$ de noretindrona, el peak de etinilestradiol disminuyó en 18 a 25\% dependiendo de la dosis de $200 \mathrm{mg}$ y $400 \mathrm{mg}$ de topiramato respectivamente. Con dosis de $400 \mathrm{mg}$ el clearance fue de $30 \%$ para el etinilestradiol y de $22 \%$ para la noretindrona; esto permite concluir que el topiramato disminuiría la eficacia de la combinación de los ACO $(15,27)$.

9) Oxacarbamazepina: Dos estudios han demostrado una marcada reducción en la concentración de etinilestradiol y levonogestrel, concluyendo que la acción de la oxicarbamazepina en la CYP3A es comparable a la de la carbamazepina. El primer estudio realizado con diferentes dosis de etinilestradiol y levonogestrel en los diferentes días del ciclo demostró que el peak medio de ambos no tuvo cambios significativos al adicionar la oxacarbamazepina. Sin embargo, la biodisponibilidad de las hormonas sexuales esteroidales fue significativamente menor con un $32 \%$ y un $48 \%$ respectivamente $(15,28)$. En otros estudios se comparó oxacarbamazepina con etinilestradiol $50 \mu \mathrm{g}$ y levonogestrel $250 \mu \mathrm{g}$. Se usó en 1 a 3 ciclos con 21 píldoras al mes; comparado con placebo, las usuarias de oxacarbamazepina tuvieron una marcada reducción en la concentración plasmática de ambos esteroides, con una reducción media de $47 \%$ para ambos $(15,29)$.

\section{OTROS MÉTODOS ANTICONCEPTIVOS HORMONALES}

Píldoras solo con progestágenos. Por las interacciones entre los antiepilépticos y la progesterona, no es confiable su utilización (30). En cuanto a la píldora del día después puede ser usada en aquellas pacientes epilépticas sin protección anticonceptiva (31).

Inyectables de medroxiprogesterona. No hay evidencia que los anticonvulsivantes que inducen las enzimas microsomales reduzcan la eficacia de 
la medroxiprogesterona, pero teóricamente lo pueden hacer, por lo que se recomienda el uso cada 10 semanas y no cada 12, esta recomendación es válida para los preparados inyectables mensuales combinados $(15,31,32,33)$.

Implantes de levonogestrel. Fueron estudiados para los subdérmicos en cápsulas, observándose que al medir los niveles hormonales de las pacientes, éstas presentaban una baja en las concentraciones del levonogestrel en las usuarias de fenitoína comparado con los controles. En los controles no hubo ningún embarazo usando el implante y si hubo dos embarazos en las usuarias de anticonvulsivantes, ambas tomando fenitoína y sus niveles de levonogestrel fueron bajos durante el período de la concepción. Además se debe destacar que el levonogestrel no altera la frecuencia de las convulsiones $(15,31,33,34,35)$.

Progestágenos intrauterinos. El uso de dispositivos intrauterinos (endoceptivo) con levonogestrel a nivel local no es afectado por la acción de las enzimas inducidas por los anticonvulsivantes. Por lo tanto las mujeres con anticonvulsivantes pueden usar este método sin riesgo aparente $(30,32)$.

\section{RECOMENDACIONES}

La evidencia indica que el uso de los ACO no tiene efectos sobre las convulsiones ya sea en su frecuencia o severidad, por lo que no se recomienda suspenderlos $(11,12)$.

Hay un número importante de anticonvulsivantes que no interactúan con los ACO como: ácido valproico, vigabatrina, lamotrigina, gabapentina, tiagabina, levotiracem, zonisamida, etosuximida y las benzodiazepinas. Sin embargo, las pacientes que toman anticonvulsivantes inductores enzimáticos como: fenobarbital, primidona, fenitoína, carbamazepina, felbamato, oxacarbamazepina y topiramato, deben incrementar las dosis de etinilestradiol a lo menos hasta $50 \mu \mathrm{g}$. Si ocurren alteraciones de sangrado intermenstrual puede ser necesario incrementar la dosis a 75 ó $100 \mu \mathrm{g}$ y advertir a las pacientes que aún utilizando estas altas dosis ellas pueden no ser garantes de no embarazarse. Sin embargo, la tasa de embarazos es de aproximadamente 7\% comparado con 15 a $20 \%$ de los métodos de barrera $(5,6,7,11,15$, $26,32,36)$. Algunos autores recomiendan el uso de un segundo método anticonceptivo en usuarias de antiepilépticos inductores de enzimas hepáticas.

Las pacientes con altas dosis de preparados con estrógenos deben recibir información del ries- go de tromboembolismo (11). Al aumentar las dosis de los ACO se debe evaluar cuidadosamente a cada paciente para evaluar el riesgo individual de esa paciente. Puede ser recomendado la monitorización de niveles hormonales al iniciar un ACO o al ver la necesidad de aumentar la dosis $(5,7,15)$.

Se recomienda el uso de protección adicional, como el uso de espermicidas o métodos de barrera asociado a los ACO, especialmente en los casos en que hay sangrado intermenstrual o irregularidad menstrual $(7,15)$. El ácido ascórbico compite con el etinilestradiol por la conjugación sulfato en la pared gastrointestinal, lo que aumenta la biodisponibilidad de las hormonas sexuales, algunos autores han demostrado hasta un $47 \%$ de incremento en la circulación de etinilestradiol después de administrar $1 \mathrm{~g}$ de vitamina $\mathrm{C}$ diaria (5, 37). Otras alternativas son el uso de dispositivos intrauterinos, endoceptivos o inyecciones de medroxiprogesterona $(15,30,32)$. En pacientes epilépticas con paridad cumplida se debe considerar la posibilidad de esterilización quirúrgica (11).

\section{BIBLIOGRAFÍA}

1. Jarrar RG, Buchhalter JR. Therapeutics in pediatric epilepsy, Part 1: The new antiepileptic drugs and the ketogenic diet. Mayo Clin Proc 2003; 78: 359.

2. Huang SH, Gambi F, Conti F, Carratelli G, Conti CM, Mastromauro I, Riccioni G, Grill A, Bellati U, Doyle RL. Antiepileptic drugs lower contraceptive sex hormone and increase the risk of unplanned pregnancies in women with epilepsy: revisited study. Int J Immunopathol Pharmacol 2003; 16(2): 181-83.

3. Guberman A. Hormonal contraception and epilepsy. Neurology 1999; 53(Suppl. 1): S38-S40.

4. Coulam CB, Annergers JF. Do anticonvulsants reduce the efficacy of oral contraceptives? Epilepsia 1979; 20: 519-25.

5. Hyman M. Sex Hormones in Stroke, Chorea, and Anticonvulsant Therapy. Seminars in Neurology 1988; 8(3): 181-6.

6. Shorvon SD, Tallis RC, Wallace HK. Antiepileptic drugs: coprescription of proconvulsant drugs and oral contraceptives: a national study of antiepileptic drug prescribing practice. J Neurol Neurosurg Psychiatry 2002; 72: 114-15.

7. Folvary N. Treatment issues for women with epilepsy. Neurol Clin North Am 2001; 19(2): 409-25.

8. Vessey $M$, Painter R, Yeates $D$. Oral contraception and epilepsy: findings in a large cohort study. Contraception 2002; 66: 77-9.

9. Chang SI, McAuley JW. Pharmacotherapeutic issues for women of chilbearing age with epilepsy. Ann Pharmacother 1998; 32: 794-801.

10. Ramsay RE, Slater JD. Effects of antiepileptic drugs on hormones. Epilepsia 1991; 32(Suppl 6): S60-67. 
11. Mattson RH, Cramer JA, Darney PD, Naftolin F. Use of oral contraceptives by women with epilepsy. JAMA 1986; 256(2): 238-40.

12. Chang SI, McAuley JW. Pharmacotherapeutic issues for women of childbearing age with epilepsy. Annals Pharmacotherapy 1998; 32(7-8): 794-801.

13. Association of the British Pharmaceutical Industry: Hallado en: www.vhn.net.

14. Yerby Mark S. Contraception, pregnancy and lactation in women with epilepsy. Bailliere's Clinical Neurology 1996; 5(4): 887-908.

15. Crawford P. Interactions between antiepileptic drugs and hormonal contraception. CNS Drugs 2002; 16(4): 263-72.

16. Diamond MP, Greene JW, Thompson JM, Van Hooydonk JE, Wentz AC. Interaction of anticonvulsants and oral contraceptives in epileptic adolescents. Contraception 1985; 31: 623-32.

17. Patsalos PN, Perucca E. Clinically important drug interactions in epilepsy: general features and interactions between antiepileptic drugs. Lancet Neurology 2003; 2: 347-56.

18. Santín J. Epilepsia en la mujer. Cuadernos de Neurología. Vol. XXV, 2001.

19. Wilkinson GR. Clearance approaches in pharmacology. Pharmacol Rev 1987; 39: 1-47.

20. Back DJ, Bates M, Bowden A. The interactions of phenobarbital and other anticonvulsants with oral contraceptive therapy. Contraception 1980; 22: 495503.

21. Crawford P, Chadwick DJ, Martín C. The interaction of phenytoin and carbamazepine with combined oral contraceptive steroids. $\mathrm{Br} J$ Clin Pharmacol 1990; 30: 892-6.

22. Crawford PM, Chadwick D, Cleland P. The lack of effect of sodium valproate on the pharmacokinetics of oral contraceptives. Contraception 1986; 33: 239.

23. Sonnen $\mathrm{AEH}$. Sodium valproate and the pill. In: Akimoto H, Kazamatsu H, Seino M. (eds). Advances in epileptology. Proceedings of the $13^{\text {th }}$ Epilepsy International Symposium. New York: Raven Press, 1982: 429-32.

24. Bartoli A, Gatti G, Cipolla G, et al. A double-blind placebo controlled study on the effect of vigabatrin on in vivo parameters of hepatic microsomal enzime induction and on the kinetics of steroid oral contraceptives in healthy female volunteers. Epilepsia 1997; 36: 702-7.

25. Holdich $\mathrm{T}$, Whiteman $\mathrm{P}$, Orme $\mathrm{M}$, et al. Effect of lamotrigine on the pharmacology of the combined oral contraceptive pill. Epilepsia 1991; 32(Suppl) 1 : 96.

26. Eldon MA, Underwood BA, Randinitis EJ, et al. Gabapentin does not interact with a contraceptive regimen of norethindrone acetate and ethinyl estradiol. Neurology 1998; 50: 1146-8.

27. Rosenfeld WE, Doce DR, Walker SA, et al. Effects of topiramate on the pharmacokinetics of an oral contraceptive containing norethindrone and ethinyl estradiol in patients with epilepsy. Epilepsia 1997; 38: 317-23.

28. Klosterkov JP, Saano V, Harring $P$, et al. Possible interaction between oxcarbazepine and an ora contraceptive. Epilepsia 1992; 33: 1149-52.

29. Fattore C, Cipolla G, Gatti G, et al. Induction of ethinylestradiol and levonorgestrel metabolism by oxacarbazepine in healthy women. Epilepsia 1999; 40: 783-7.

30. Mehta DK (ed). British National Formulary-BNF40, September 2000. London: British Medical Association and the Royal Pharmaceutical Society of Great Britain, 2000.

31. Brodie MJ, Frech JA. Management of epilepsy in adolescents and adults. Lancet 2000; 356: 323-29.

32. Betts T, Crawford P. Women and epilepsy. London: Martin Dunitz, 1998.

33. Yasser Y. El-Sayed. Obstetric and gynecologic care of women with epilepsy. Epilepsia 1998; 39(Suppl, 8): S17-S25.

34. Krauss GL, Brandt J, Campbell M, et al. Antiepileptic medication and oral contraceptive interactions: a national survey of neurologists and obstetricians. Neurology 1996; 46: 1534-9.

35. Haukkamaa M. Contraception by Norplant subdermal capsules is not reliable in epileptic patients on anticonvulsant therapy. Contraception 1986; 33: 55965.

36. Trussell J, Kost K. A guide to interpreting contraceptive efficacy studies. Obstet Gynacol 1990; 76: 55867.

37. Back D, Breckinridge A, Maclver M, et al. The interaction of ethinylestradiol with ascorbic acid in man. Br Med J 1981; 282: 1516. 